\title{
ON THE CARTIER DUALITY OF CERTAIN FINITE GROUP SCHEMES OF TYPE $\left(p^{n}, \ldots, p^{n}\right)$
}

\author{
By
}

\author{
Nobuhiro AKI
}

\begin{abstract}
In this paper, we determine the Cartier dual of certain finite group schemes of type $\left(p^{n}, \ldots, p^{n}\right)$, restricting ourselves to positive characteristic $p$ case. They are given by the kernel of certain endomormphisms of the fibre product $W_{l, A} \times_{\operatorname{Spec} A} \cdots \times_{\operatorname{Spec} A} W_{l, A}$ of the group scheme of Witt vectors of the length $l$. Moreover we can treat the kernel of the endomorphism of a type $F^{n}+a_{1} F^{n-1}$ $+\cdots+\boldsymbol{a}_{n}: W_{l, A} \rightarrow W_{l, A}$ as our special class, where $F$ is the Frobenius endomorphism and $\boldsymbol{a}_{k}(k=1, \ldots, n)$ are suitable Witt vectors.
\end{abstract}

\section{Introduction}

Throughout this paper, we denote by $p$ a prime number. Let $A$ be a commutative unitary ring of characteristic $p$. For a group scheme $G$ over $A$, we denote by $\hat{G}$ the formal completion of $G$ along the zero section. Our argument is expanded on the group schemes introduced by T. Sekiguchi and N. Suwa [SS2, Theorem 3.2 and Theorem 3.3]

$$
\begin{aligned}
\mathscr{E}_{n}:=\operatorname{Spec} A & {\left[X_{0}, X_{1}, \ldots, X_{n-1}, \frac{1}{1+\lambda_{1} X_{0}},\right.} \\
& \left.\frac{1}{D_{1}\left(X_{0}\right)+\lambda_{2} X_{1}}, \frac{1}{D_{n-1}\left(X_{0}, \ldots, X_{n-2}\right)+\lambda_{n} X_{n-1}}\right] .
\end{aligned}
$$

These group schemes are constructed inductively by the following extensions;

$$
\mathscr{E}_{1}=\mathscr{G}^{\left(\lambda_{1}\right)}=\operatorname{Spec} A\left[X_{0}, \frac{1}{1+\lambda_{1} X_{0}}\right]
$$

Received March 8, 2010.

Revised July 9, 2010. 


$$
\begin{array}{ccc}
0 \longrightarrow \mathscr{G}^{\left(\lambda_{2}\right)} \longrightarrow \mathscr{E}_{2}=\operatorname{Spec} A\left[X_{0}, X_{1}, \frac{1}{1+\lambda_{1} X_{0}}, \frac{1}{D_{0}\left(X_{0}\right)+\lambda_{2} X_{1}}\right] & \longrightarrow \mathscr{E}_{1} \longrightarrow 0 \\
0 \longrightarrow \mathscr{G}^{\left(\lambda_{3}\right)} \longrightarrow & \longrightarrow \mathscr{E}_{2} \longrightarrow 0 \\
0 & \vdots & \\
0 \mathscr{G}^{\left(\lambda_{n-1}\right)} \longrightarrow & \mathscr{E}_{n} & \longrightarrow \mathscr{E}_{n-1} \longrightarrow 0 .
\end{array}
$$

Here, $\lambda_{1}, \lambda_{2}, \ldots, \lambda_{n}$ are elements of $A$, and $D_{i}\left(X_{0}, X_{1}, \ldots, X_{n-1}\right)$ 's are given as elements of $\operatorname{Hom}_{A / \lambda_{i}}\left(\hat{\mathscr{E}}_{i-1}, \hat{\mathbf{G}}_{m, A / \lambda_{i}}\right)$. For deciding $D_{i}$ 's more explicitly, they introduced the endomorphism $U^{n}: W_{A}^{n} \rightarrow W_{A}^{n}$ on the fibre product space of group schemes of Witt vectors, and showed the canonical isomorphism;

$$
\operatorname{Ker}\left[U^{n}: W_{A}(A)^{n} \rightarrow W_{A}(A)^{n}\right] \simeq \operatorname{Hom}\left(\hat{\mathscr{E}}_{n}, \hat{\mathbf{G}}_{m, A}\right) .
$$

Moreover they also showed the canonical isomorphism;

$$
\operatorname{Coker}\left[U^{n}: W_{A}(A)^{n} \rightarrow W_{A}(A)^{n}\right] \simeq H_{0}^{2}\left(\hat{\mathscr{E}}_{n}, \hat{\mathbf{G}}_{m, A}\right),
$$

where $H_{0}^{2}\left(\hat{\mathscr{E}}_{n}, \hat{\mathbf{G}}_{m, A}\right)$ means the Hochschild cohomology groups.

By these results, the homomorphism $D_{i} \in \operatorname{Hom}_{A / \lambda_{i}}\left(\hat{\mathscr{E}}_{i-1}, \hat{\mathbf{G}}_{m, A / \lambda_{i}}\right)$ is given by an element in $\operatorname{Ker}\left[U^{i-1}: W_{A / \lambda_{i}}\left(A / \lambda_{i}\right)^{i-1} \rightarrow W_{A / \lambda_{i}}\left(A / \lambda_{i}\right)^{i-1}\right]$. The group scheme structure of $\mathscr{E}_{n}$ is given by the one which makes the morphism

$$
\alpha^{(n)}: \mathscr{E}_{n} \rightarrow \mathbf{G}_{m, A}^{n},
$$

defined by

$$
\left(x_{0}, x_{1}, \ldots, x_{n-1}\right) \mapsto\left(1+\lambda_{1} x_{0}, D_{1}\left(x_{0}\right)+\lambda_{2} x_{1}, \ldots, D_{n-1}\left(x_{0}, \ldots, x_{n-2}\right)+\lambda_{n} x_{n-1}\right),
$$

a homomorphism of group schemes. Hereafter let $l$ be a positive integer. For a given group scheme $\mathscr{E}_{n}$ such as above, if we take the $p^{l}$-th power of the data defining $\mathscr{E}_{n}$, then those data defines a group scheme $\mathscr{E}_{n}^{p}$;

$$
\begin{aligned}
\mathscr{E}_{n}^{p^{l}}:=\operatorname{Spec} A[ & {\left[X_{0}, X_{1}, \ldots, X_{n-1}, \frac{1}{1+\lambda_{1}^{p^{l}} X_{0}},\right.} \\
& \left.\frac{1}{D_{1}^{\prime}\left(X_{0}\right)+\lambda_{2}^{p^{l}} X_{1}}, \frac{1}{D_{n-1}^{\prime}\left(X_{0}, \ldots, X_{n-2}\right)+\lambda_{n}^{p^{l}} X_{n-1}}\right] .
\end{aligned}
$$

The group scheme structure of $\mathscr{E}_{n}^{p^{l}}$ is given by the one which makes the morphism

$$
\alpha^{(n)^{\prime}}: \mathscr{E}_{n}^{p^{l}} \rightarrow \mathbf{G}_{m, A}^{n}
$$


defined by

$$
\left(x_{0}, x_{1}, \ldots, x_{n-1}\right) \mapsto\left(1+\lambda_{1}^{p^{l}} x_{0}, D_{1}^{\prime}\left(x_{0}\right)+\lambda_{2}^{p^{l}} x_{1}, \ldots, D_{n-1}^{\prime}\left(x_{0}, \ldots, x_{n-2}\right)+\lambda_{n}^{p^{l}} x_{n-1}\right),
$$

a homomorphism. And this satisfies the following commutative diagram;

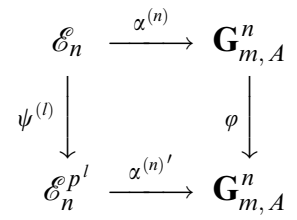

where $\varphi$ is given by $\varphi\left(t_{0}, \ldots, t_{n-1}\right)=\left(t_{0}^{p^{l}}, \ldots, t_{n-1}^{p^{l}}\right)$ and $\psi^{(l)}$ is isogeny defined by $\psi^{(l)}\left(x_{0}, \ldots, x_{n-1}\right)=\left(x_{0}^{p^{l}}, \ldots, x_{n-1}^{p^{l}}\right)$. Then the kernel $N_{l}=\operatorname{Ker} \psi^{(l)}$ is given explicity by

$$
N_{l}=\operatorname{Spec} A\left[X_{0}, \ldots, X_{n-1}\right] /\left(X_{0}^{p^{l}}, \ldots, X_{n-1}^{p^{l}}\right),
$$

and we have the exact sequence;

$$
0 \longrightarrow N_{l} \stackrel{l}{\longrightarrow} \mathscr{E}_{n} \stackrel{\psi^{(l)}}{\longrightarrow} \mathscr{E}_{n}^{p^{l}} \longrightarrow 0
$$

Note that the group scheme sturucture of $N_{l}$ is the one induced from $\mathscr{E}_{n}$. In our argument, the important thing is that we can identify the finite group scheme $N_{l}$ with the completion $\hat{N}_{l}$, because $X_{i}$ 's are nilpotents in the coordinate ring of $N_{l}$, and we can consider the exact sequence;

$$
0 \longrightarrow N_{l} \stackrel{l}{\longrightarrow} \hat{\mathscr{E}}_{n} \stackrel{\psi^{(l)}}{\longrightarrow} \hat{\mathscr{E}}_{n}^{p^{l}} \longrightarrow 0
$$

By means of the definition of the endomorphism

$$
U^{n}: W_{A}^{n} \rightarrow W_{A}^{n}
$$

it induces an endomorphism

$$
U_{l}^{n}: W_{l, A}^{n} \rightarrow W_{l, A}^{n}
$$

which makes the following commutative diagram;

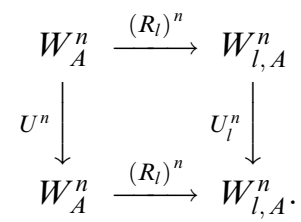

Under these notations, our first main result is given as follows; 
THEOREM 1. Assum that $A$ is a commutative unitary ring of chracteritic p. Then the Cartier dual of $N_{l}$ is canonically isomorphic to $\operatorname{Ker}\left[U_{l}^{n}: W_{l, A}^{n} \rightarrow W_{l, A}^{n}\right]$.

Oort-Tate $[\mathrm{OT}]$ gave the result of Theorem 1 in the case of $l=n=1$. Next M. Amano [A] proved Theorem 1 for any $l$ and $n=1$, and N. Aki and M. Amano [AA] proved Theorem 1 for any $l$ and $n=2$ by using the deformations of Artin-Hasse exponential series. We prove Theorem 1 in the general case by generalizing the argument in the previous paper [AA].

Let $K$ be a perfect field of characteritic $p$. Then we have Dieudonné ring $\mathbf{D}_{K}$ and the isomorphism $\mathbf{D}_{K} / \mathbf{D}_{K} V^{l} \simeq \operatorname{Hom}\left(W_{l, K}, W_{l, K}\right)$. ([DG, p. 550].) From this point of view, $F^{n}+\boldsymbol{a}_{1} F^{n-1}+\cdots+\boldsymbol{a}_{n}$ is an element of $\mathbf{D}_{K} / \mathbf{D}_{K} V^{l}$ for Witt vectors $\boldsymbol{a}_{1}, \ldots, \boldsymbol{a}_{n} \in W_{l, A}$. In Section 6 , we give an isomorphism;

$$
\operatorname{Ker}\left[U_{l}^{n}: W_{l, A}^{n} \rightarrow W_{l, A}^{n}\right] \simeq \operatorname{Ker}\left[F^{n}+\boldsymbol{a}_{1} F^{n-1}+\cdots+\boldsymbol{a}_{n}: W_{l, A} \rightarrow W_{l, A}\right],
$$

for some special type of Witt vectors $\boldsymbol{a}_{1}, \ldots, \boldsymbol{a}_{n} \in W_{l, A}$, and we give the second asertion;

THEOREM 2. If we choose the base ring $A$ of characteritic $p$ and the group scheme $\mathscr{E}_{n}$ suitably, then the Cartier dual of $N_{l}$ is canonically isomorphic to $\operatorname{Ker}\left[F^{n}+\boldsymbol{a}_{1} F^{n-1}+\cdots+\boldsymbol{a}_{n}: W_{l, A} \rightarrow W_{l, A}\right]$, where for each $1 \leq k \leq n, \boldsymbol{a}_{k}$ is Witt vectors given by $\boldsymbol{a}_{k}=\sum_{n \geq i_{1}>i_{2} \cdots>i_{k} \geq 1}(-1)^{k}\left[\prod_{j=1}^{k} \lambda_{i_{k}}^{(p-1) p^{n-i_{j}-(j-1)}}\right]$, and $\left[\lambda_{i_{k}}\right]$ is the Teichmüller lifting $\left(\lambda_{i_{k}}, 0, \ldots\right) \in W(A)$ of $\lambda_{i_{k}} \in A$.

The contents of this paper is as follows. The next two sections are devoted to give the definitions and some reviews of properties of Witt vectors, the deformed Artin-Hasse exponential series and the group schemes $\mathscr{E}_{n}$ and $\mathscr{E}_{n}{ }^{l}$. In Section 5 and Section 6 we give the proofs of Theorem 1 and Theorem 2 .

\section{Notations}

$\mathbf{G}_{m, A}$ : the multiplicative group scheme over $A$

$W_{n, A}$ : the group scheme of Witt vectors of length $n$ over $A$

$W_{A}$ : the group scheme of Witt vectors over $A$

$\hat{\mathbf{G}}_{m, A}$ : the multiplicative formal group scheme over $A$

$\hat{W}_{n, A}$ : the formal group scheme of Witt vectors of length $n$ over $A$

$\hat{W}_{A}$ : the formal group scheme of Witt vectors over $A$

$F$ : the Frobenius of endomorphism of $W_{A}$

$V$ : the Verschiebung endomorphism of $W_{A}$ 
$R_{n}$ : the restriction homomorphism of $W_{A}$ to $W_{n, A}$

$[\lambda]$ : the Teichmüller lifting $(\lambda, 0, \ldots) \in W(A)$ of $\lambda \in A$

$\boldsymbol{a}^{(p)}:=\left(a_{0}^{p}, a_{1}^{p}, \ldots\right)(=F(\boldsymbol{a})) \quad\left(\boldsymbol{a}=\left(a_{0}, a_{1}, \ldots\right) \in W(A)\right)$

$F^{(\lambda)}:=F-\left[\lambda^{p-1}\right]$

$\mathbf{X}:=\left(X_{0}, X_{1}, \ldots\right) \quad$ (a sequence of variables)

$\mathbf{Y}:=\left(Y_{0}, Y_{1}, \ldots\right)$ (a sequence of variables)

\section{Acknowledgments}

The author expresses his gratitude to Professor Tsutomu Sekiguchi for his kind advice, suggestions and his careful reading of the manuscript. He thanks also Michio Amano for stimulative conversaitions.

\section{Witt Vectors}

In this short section we recall necessary facts on Witt vectors for this paper. For details, see [DG, Chap. V] or [HZ, Chap. III].

2.1. Let $\mathbf{X}=\left(X_{0}, X_{1}, \ldots\right)$ be a sequence of variables. For each $n \geq 0$, we denote by $\Phi_{n}(\mathbf{X})=\Phi_{n}\left(X_{0}, X_{1}, \ldots, X_{n}\right)$ the Witt polynomial

$$
\Phi_{n}(\mathbf{X})=X_{0}^{p^{n}}+p X_{1}^{p^{n-1}}+\cdots+p^{n} X_{n}
$$

in $\mathbf{Z}[\mathbf{X}]=\mathbf{Z}\left[X_{0}, X_{1}, \ldots\right]$. Let $W_{n, \mathbf{Z}}=\operatorname{Spec} \mathbf{Z}\left[X_{0}, X_{1}, \ldots, X_{n-1}\right]$ be the $n$-dimentional affine space over $\mathbf{Z}$. We define a morphism (the so-called Phantom map) $\Phi^{(n)}$ by

$$
\Phi^{(n)}: W_{n, \mathbf{Z}} \rightarrow \mathbf{A}_{\mathbf{Z}}^{n} ; \quad \boldsymbol{x} \mapsto\left(\Phi_{0}(\boldsymbol{x}), \Phi_{1}(\boldsymbol{x}), \ldots, \Phi_{n-1}(\boldsymbol{x})\right) .
$$

Note that $W_{n, \mathbf{Z}}$ has the ring so that $\Phi^{(n)}$ becomes a ring scheme homomorphism, when $\mathbf{A}_{\mathbf{Z}}^{n}$ is regarded as a ring scheme by coordinate-wise addition and multiplication.

2.2. The Verschiebung homomorphism $V$ is defined by

$$
V: W(A) \rightarrow W(A) ; \quad \boldsymbol{x}=\left(x_{0}, x_{1}, \ldots\right) \mapsto V(\boldsymbol{x})=\left(0, x_{1}, x_{2}, \ldots\right) .
$$

The restriction homorphism $R_{n}$ is defined by

$$
R_{n}: W(A) \rightarrow W_{n}(A) ; \quad \boldsymbol{x}=\left(x_{0}, x_{1}, \ldots\right) \mapsto \boldsymbol{x}_{n}=\left(x_{0}, x_{1}, \ldots, x_{n-1}\right) .
$$

We define a morphism $F: W_{n}(A) \rightarrow W_{n-1}(A)$ by

$$
\Phi_{i}(F \boldsymbol{x})=\Phi_{i+1}(\boldsymbol{x})
$$


for $\boldsymbol{x} \in W_{n}(A)$. If $A$ is of characteristic $p, F$ is noting but the usual Frobenius endomorphism. For $\lambda \in A,[\lambda]$ and $F^{(\lambda)}$ denote the Teichmüller lifting $[\lambda]=$ $(\lambda, 0, \ldots) \in W(A)$ and the endomorphism $F-\left[\lambda^{p-1}\right]$ of $W(A)$, respectively. For $\boldsymbol{a}=\left(a_{0}, a_{1}, \ldots\right) \in W(A)$, we also define a morphism $T_{\boldsymbol{a}}: W(A) \rightarrow W(A)$ by

$$
\Phi_{n}\left(T_{\boldsymbol{a}} \boldsymbol{x}\right)=a_{0}^{p^{n}} \Phi_{n}(\boldsymbol{x})+p a_{1}^{p^{n-1}} \Phi_{n-1}(\boldsymbol{x})+\cdots+p^{n} a_{n} \Phi_{0}(\boldsymbol{x})
$$

for $\boldsymbol{x} \in W(A)$. Then it is known that this morphism satisfies the formula $T_{\boldsymbol{a}}=\sum_{k \geq 0} V^{k} \cdot\left[a_{k}\right]$. (cf. [SS2, Chap. 4, p. 20].)

\section{Deformed Artin-Hasse Exponential Series}

In this short section we recall nececssary facts on the deformed Artin-Hasse exponential series for this paper.

3.1. The Artin-Hasse exponential series $E_{p}(X)$ is given by

$$
E_{p}(X)=\exp \left(\sum_{r \geq 0} \frac{X^{p^{r}}}{p^{r}}\right) \in \mathbf{Z}_{(p)}[[X]] .
$$

We define a formal power series $E_{p}(U, \lambda ; X)$ in $\mathbf{Q}[U, \lambda][[X]]$ by

$$
\left.E_{p}(U, \Lambda ; X)=(1+\Lambda X)^{U / \Lambda} \prod_{k=1}^{\infty}\left(1+\Lambda^{p^{k}} X^{p^{k}}\right)^{\left(1 / p^{k}\right)\left((U / \Lambda)^{p^{k}}-(U / \Lambda)^{p^{k-1}}\right.}\right)
$$

As in [SS1, Corollrary 2.5] or [SS2, Lemma 4.8], we see that this formal power series $E_{p}(U, \lambda ; X)$ is integral over $\mathbf{Z}_{(p)}$.

Let $A$ be a $\mathbf{Z}_{(p)}$-algebra. Let $\lambda \in A$ and $\boldsymbol{v}=\left(v_{0}, v_{1}, \ldots\right) \in W(A)$. We define a formal power series $E_{p}(\boldsymbol{v}, \lambda ; X)$ in $A[[X]]$ by

$$
\begin{aligned}
E_{p}(\boldsymbol{v}, \lambda ; X) & =\prod_{k=0}^{\infty} E_{p}\left(v_{k}, \lambda^{p^{k}} ; X^{p^{k}}\right) \\
& =(1+\lambda X)^{v_{0} / \lambda} \prod_{k=1}^{\infty}\left(1+\lambda^{p^{k}} X^{p^{k}}\right)^{\left(1 / p^{k} \lambda^{p^{k}}\right) \Phi_{k-1}\left(F^{(\lambda)} \boldsymbol{v}\right)} .
\end{aligned}
$$

Moreover we define a formal power series $F_{p}(\boldsymbol{v}, \lambda ; X, Y)$ as follows;

$$
F_{p}(\boldsymbol{v}, \lambda ; X, Y)=\prod_{k=1}^{\infty}\left(\frac{\left(1+\lambda^{p^{k}} X^{p^{k}}\right)\left(1+\lambda^{p^{k}} Y^{p^{k}}\right)}{1+\lambda^{p^{k}}(X+Y+\lambda X Y)^{p^{k}}}\right)^{\left(1 / p^{k} \lambda^{p^{k}}\right) \Phi_{k-1}(\boldsymbol{v})} .
$$


As in [SS1, Lemma 2.16] or [SS2, Lemma 4.9], we see that the formal power series $F_{p}(\boldsymbol{v}, \lambda ; X, Y)$ satisfies the formula;

$$
\frac{E_{p}(\boldsymbol{v}, \lambda ; X) E_{p}(\boldsymbol{v}, \lambda ; Y)}{E_{p}(\boldsymbol{v}, \lambda ; X+Y+\lambda X Y)}=F_{p}\left(F^{(\lambda)} \boldsymbol{v}, \lambda ; X, Y\right) .
$$

\section{Definitions of the Group Schemes $\mathscr{E}_{n}$ and $\mathscr{E}_{n}{ }^{l}$}

We review here the group schemes $\mathscr{E}_{n}$ briefly from T. Sekiguchi and N. Suwa [SS2, Theorem 3.3].

4.1. Let $A$ be a $\mathbf{Z}_{(p)}$-algebra and $\lambda, \lambda_{1}, \ldots, \lambda_{n}$ be non-zero elements of $A$. For a vector $\overline{\boldsymbol{a}}$ of $W(A / \lambda)$, we denote by $\boldsymbol{a} \in W(A)$ a representative of $\overline{\boldsymbol{a}}$. Note that the formal completion $\hat{W}$ is characterized as a functor given by;

$$
\hat{W}(A)=\left\{\left(a_{0}, a_{1}, \ldots\right) \in W(A) \mid a_{i} \text { are nilpotents and } a_{i}=0 \text { for almost all } i\right\} .
$$

We choose Witt vectors

$$
\overline{\boldsymbol{a}}^{i}=\left(\overline{\boldsymbol{a}}_{j}^{i}\right)_{1 \leqq j \leqq i} \in \operatorname{Ker}\left[U^{i}: \hat{W}\left(A / \lambda_{i+1}\right)^{i} \rightarrow \hat{W}\left(A / \lambda_{i+1}\right)^{i}\right]
$$

inductively by the following recursive conditions;

$$
\begin{gathered}
U^{1}:=F^{\left(\lambda_{1}\right)}, \quad \overline{\boldsymbol{a}}^{1}:=\overline{\boldsymbol{a}}_{1}^{1} \in \operatorname{Ker}\left[U^{1}: \hat{W}\left(A / \lambda_{2}\right) \rightarrow \hat{W}\left(A / \lambda_{2}\right)\right] \\
\boldsymbol{b}_{1}^{2}:=\frac{1}{\lambda_{2}} F^{\left(\lambda_{1}\right)} \boldsymbol{a}_{1}^{1}, \quad U^{2}:=\left(\begin{array}{cc}
F^{\left(\lambda_{1}\right)} & -T_{\boldsymbol{b}_{1}^{2}} \\
0 & F^{\left(\lambda_{2}\right)}
\end{array}\right),
\end{gathered}
$$

and for $k \geqq 2$, we choose

$$
\overline{\boldsymbol{a}}^{k}:=\left(\overline{\boldsymbol{a}}_{j}^{k}\right)_{1 \leqq j \leqq k} \in \operatorname{Ker}\left[U^{k}: \hat{W}\left(A / \lambda_{k+1}\right)^{k} \rightarrow \hat{W}\left(A / \lambda_{k+1}\right)^{k}\right],
$$

and we define

$$
\begin{aligned}
\boldsymbol{b}_{j}^{k+1} & :=\frac{1}{\lambda_{k+1}}\left(F^{\left(\lambda_{j}\right)} \boldsymbol{a}_{j}^{k}-\sum_{l=j+1}^{k} T_{\boldsymbol{b}_{j}^{l}} \boldsymbol{a}_{l}^{k}\right) \quad 1 \leqq j \leqq k-1 \\
\boldsymbol{b}_{k}^{k+1} & :=\frac{1}{\lambda_{k+1}} F^{\left(\lambda_{k}\right)} \boldsymbol{a}_{k}^{k} \\
U^{k+1} & :=\left(\begin{array}{ccccc}
F^{\left(\lambda_{1}\right)} & -T_{\boldsymbol{b}_{1}^{2}} & \cdots & \cdots & -T_{\boldsymbol{b}_{1}^{k+1}} \\
0 & F^{\left(\lambda_{2}\right)} & -T_{\boldsymbol{b}_{2}^{3}} & \cdots & -T_{\boldsymbol{b}_{2}^{k+1}} \\
0 & \cdots & & & -T_{\boldsymbol{b}_{k}^{k+1}} \\
0 & & \ldots & & F^{\left(\lambda_{k+1}\right)}
\end{array}\right)
\end{aligned}
$$


Moreover we define formal power series $D_{k}\left(X_{0}, X_{1}, \ldots, X_{k-1}\right)(k \geqq 1)$ by

$$
\begin{aligned}
D_{0} & =1 \\
D_{1}\left(X_{0}\right) & =E_{p}\left(\overline{\boldsymbol{a}}_{1}^{1}, \lambda_{1} ; X_{0}\right)
\end{aligned}
$$

and for $k \geqq 1$

$$
\begin{aligned}
D_{k+1}\left(X_{0}, X_{1}, \ldots, X_{k}\right) & =E_{p}\left(\overline{\boldsymbol{a}}^{k+1},\left(\lambda_{i}\right)_{1 \leqq i \leqq k+1} ; X_{0}, X_{1}, \ldots, X_{k}\right) \\
& :=\prod_{i=1}^{k+1} E_{p}\left(\overline{\boldsymbol{a}}_{i}^{k+1}, \lambda_{i} ; \frac{X_{i-1}}{D_{i-1}\left(X_{0}, X_{1}, \ldots, X_{i-2}\right)}\right) .
\end{aligned}
$$

We put

$$
\begin{aligned}
\mathscr{E}_{n}:=\operatorname{Spec} A\left[X_{0}, X_{1}, \ldots, X_{n-1}, \frac{1}{1+\lambda_{1} X_{0}},\right. \\
\\
\left.\quad \frac{1}{D_{1}\left(X_{0}\right)+\lambda_{2} X_{1}}, \ldots, \frac{1}{D_{n-1}\left(X_{0}, \ldots, X_{n-2}\right)+\lambda_{n} X_{n-1}}\right] .
\end{aligned}
$$

Then by [SS2, Theorem 4.16 and Theorem 3.3], $\mathscr{E}_{n}$ becomes a group scheme and

$$
D_{i} \in \operatorname{Hom}_{A / \lambda_{i+1}}\left(\mathscr{E}_{n} \otimes_{A} A / \lambda_{i+1}, \mathbf{G}_{A / \lambda_{i+1}}\right), \quad \text { for } i=1, \ldots, n-1 .
$$

4.2. In this subsection, let $A$ be of characteristic $p$ and $\lambda_{1}, \ldots, \lambda_{n} \in A$. We will define a group scheme denoted by $\mathscr{E}_{n}^{p^{l}}$. Let $l$ be positive interger and $\left(\lambda_{i}^{p^{l}}\right)_{1 \leqq i \leqq k+1}$ be elements of $A$. We define Witt vectors inductively by the following recursive condisions.

For $\overline{\boldsymbol{a}}_{1}^{1} \in \operatorname{Ker} U^{1}$, we have a relation

$$
F^{\left(\lambda_{1}\right)}\left(\boldsymbol{a}_{1}^{1}\right) \equiv 0 \bmod \lambda_{2} .
$$

So we have also the following relation

$$
F^{\left(\lambda_{1}^{p^{l}}\right)}\left(\left(\boldsymbol{a}_{1}^{1}\right)^{\left(p^{l}\right)}\right) \equiv 0 \bmod \lambda_{2}^{p^{l}} .
$$

We put $\overline{\mathbf{A}}_{1}^{1}:=\overline{\boldsymbol{a}}_{1}^{1\left(p^{l}\right)}$, then $\overline{\mathbf{A}}_{1}^{1} \in \operatorname{Ker} F^{\left(\lambda_{1}^{p^{l}}\right)}$. We define $\left(U^{1}\right)^{\prime}:=F^{\left(\lambda_{1}^{p^{l}}\right)}$. Then we have the following congruences;

$$
\overline{\mathbf{A}}^{1}:=\overline{\mathbf{A}}_{1}^{1} \in \operatorname{Ker}\left[\left(U^{1}\right)^{\prime}: \hat{W}\left(A / \lambda_{2}^{p^{l}}\right) \rightarrow \hat{W}\left(A / \lambda_{2}^{p^{l}}\right)\right] .
$$

For $\left(\overline{\boldsymbol{a}}_{i}^{k}\right)_{1 \leqq i \leqq k} \in \operatorname{Ker} U^{k}$, we have following equations; 
On the Cartier duality of certain finite group schemes

$$
\begin{array}{r}
F^{\left(\lambda_{1}\right)} \boldsymbol{a}_{1}^{k}-T_{\boldsymbol{b}_{1}^{2}} \boldsymbol{a}_{2}^{k}-\cdots-T_{\boldsymbol{b}_{1}^{2}} \boldsymbol{a}_{2}^{k} \equiv 0 \bmod \lambda_{k+1} \\
F^{\left(\lambda_{2}\right)} \boldsymbol{a}_{2}^{k}-\cdots-T_{\boldsymbol{b}_{2}^{k}} \boldsymbol{a}_{k}^{k} \equiv 0 \bmod \lambda_{k+1} \\
\vdots \\
F^{\left(\lambda_{k}\right)} \boldsymbol{a}_{k}^{k} \equiv 0 \bmod \lambda_{k+1}
\end{array}
$$

and

$$
\begin{aligned}
\left(F^{\left(\lambda_{1}\right)} \boldsymbol{a}_{1}^{k}-T_{\boldsymbol{b}_{1}^{2}} \boldsymbol{a}_{2}^{k}-\cdots-T_{\boldsymbol{b}_{1}^{2}} \boldsymbol{a}_{2}^{k}\right)^{p^{l}} & \equiv 0 \bmod \lambda_{k+1}^{p^{l}} \\
\left(F^{\left(\lambda_{2}\right)} \boldsymbol{a}_{2}^{k}-\cdots-T_{\boldsymbol{b}_{2}^{k}} \boldsymbol{a}_{k}^{k}\right)^{p^{l}} & \equiv 0 \bmod \lambda_{k+1}^{p^{l}} \\
\vdots & \\
\left(F^{\left(\lambda_{k}\right)} \boldsymbol{a}_{k}^{k}\right)^{p^{l}} & \equiv 0 \bmod \lambda_{k+1}^{p^{l}} .
\end{aligned}
$$

Moreover we have the following equations;

$$
\begin{gathered}
F^{\left(\lambda_{1}^{p^{l}}\right)}\left(\boldsymbol{a}_{1}^{k}\right)^{\left(p^{l}\right)}-\cdots-T_{\boldsymbol{b}_{2}^{k\left(p^{l}\right)}}\left(\boldsymbol{a}_{k}^{k}\right)^{\left(p^{l}\right)} \equiv 0 \bmod \lambda_{k+1}^{p^{l}} \\
F^{\left(\lambda_{2}^{p^{l}}\right)}\left(\boldsymbol{a}_{2}^{k}\right)^{\left(p^{l}\right)}-\cdots-T_{\boldsymbol{b}_{2}^{k\left(p^{l}\right)}}\left(\boldsymbol{a}_{k}^{k}\right)^{\left(p^{l}\right)} \equiv 0 \bmod \lambda_{k+1}^{p^{l}} \\
\vdots \\
F^{\left(\lambda_{k}\right)^{p^{l}}}\left(\boldsymbol{a}_{k}^{k}\right)^{\left(p^{l}\right)} \equiv 0 \bmod \lambda_{k+1}^{p^{l}} .
\end{gathered}
$$

For $k \geqq 2$ we define

$$
\begin{aligned}
\left(U^{2}\right)^{\prime} & :=\left(\begin{array}{cc}
F^{\left(\lambda_{1}^{p^{l}}\right)} & -T_{\mathbf{B}_{1}^{2}} \\
0 & F^{\left(\lambda_{2}^{p^{l}}\right)}
\end{array}\right) \\
\mathbf{B}_{1}^{2} & :=\frac{1}{\lambda_{2}^{p^{l}}} F^{\left(\lambda_{1}^{p^{l}}\right)} \mathbf{A}_{1}^{1}=\left(\frac{1}{\lambda_{2}} F^{\left(\lambda_{1}\right)} \boldsymbol{a}_{1}^{1}\right)^{p^{l}}=\overline{\boldsymbol{b}}_{1}^{2\left(p^{l}\right)} \\
\mathbf{B}_{j}^{k+1} & :=\frac{1}{\lambda_{k+1}}\left(F^{\left(\lambda_{j}^{p^{l}}\right)} \mathbf{A}_{j}^{k}-\sum_{l=j+1}^{k} T_{\mathbf{B}_{j}^{l}} \mathbf{A}_{l}^{k}\right) \quad 1 \leqq j \leqq k-1 \\
\mathbf{B}_{k}^{k+1} & :=\frac{1}{\lambda_{k+1}^{p^{l}}} F^{\left(\lambda_{k}^{p^{l}}\right)} \mathbf{A}_{k}^{k}
\end{aligned}
$$




$$
\left(U^{k+1}\right)^{\prime}:=\left(\begin{array}{ccccc}
F^{\left(\lambda_{1}^{p^{l}}\right)} & -T_{\mathbf{B}_{1}^{2}} & \cdots & \cdots & -T_{\mathbf{B}_{1}^{k+1}} \\
0 & F^{\left(\lambda_{2}^{p^{l}}\right)} & -T_{\mathbf{B}_{2}^{3}} & \cdots & -T_{\mathbf{B}_{2}^{k+1}} \\
0 & \cdots & & & -T_{\mathbf{B}_{k}^{k+1}} \\
0 & & \cdots & & F^{\left(\lambda_{k+1}^{p^{l}}\right)}
\end{array}\right) .
$$

For $k \geqq 2$ we put

$$
\overline{\mathbf{A}}^{k}:=\left(\overline{\mathbf{A}}_{j}^{k}\right)_{1 \leqq j \leqq k}=\left(\overline{\boldsymbol{a}}_{j}^{k\left(p^{l}\right)}\right)_{1 \leqq j \leqq k} .
$$

And for $k \geqq 2$, we have relations

$$
\overline{\mathbf{A}}^{k}=\left(\overline{\mathbf{A}}_{j}^{k}\right)_{1 \leqq j \leqq k} \in \operatorname{Ker}\left[\left(U^{k}\right)^{\prime}: \hat{W}\left(A / \lambda_{k+1}^{p^{l}}\right)^{k} \rightarrow \hat{W}\left(A / \lambda_{k+1}^{p^{l}}\right)^{k}\right] .
$$

So we define formal power series $D_{k}^{\prime}\left(X_{0}, X_{1}, \ldots, X_{k-1}\right)(k \geqq 1)$ by

$$
\begin{aligned}
D_{0}^{\prime} & =1 \\
D_{1}^{\prime}\left(X_{0}\right) & =E_{p}\left(\overline{\mathbf{A}}_{1}^{1}, \lambda_{1}^{p^{\prime}} ; X_{0}\right)
\end{aligned}
$$

and for $k \geqq 1$

$$
\begin{aligned}
D_{k+1}^{\prime}\left(X_{0}, X_{1}, \ldots, X_{k}\right) & =E_{p}\left(\overline{\mathbf{A}}^{k+1},\left(\lambda_{i}^{p^{l}}\right)_{1 \leqq i \leqq k+1} ; X_{0}, X_{1}, \ldots, X_{k}\right) \\
& :=\prod_{i=1}^{k+1} E_{p}\left(\overline{\mathbf{A}}_{i}^{k+1}, \lambda_{i}^{p^{l}} ; \frac{X_{i-1}}{D_{i-1}^{\prime}\left(X_{0}, X_{1}, \ldots, X_{i-2}\right)}\right)
\end{aligned}
$$

Then we have a group scheme;

$$
\begin{aligned}
\operatorname{Spec} A[ & X_{0}, X_{1}, \ldots, X_{n-1}, \frac{1}{1+\lambda_{1}^{p^{l}} X_{0}}, \\
& \left.\frac{1}{D_{1}^{\prime}\left(X_{0}\right)+\lambda_{2}^{p^{l}} X_{1}}, \ldots, \frac{1}{D_{n-1}^{\prime}\left(X_{0}, \ldots, X_{n-2}\right)+\lambda_{n}^{p^{l}} X_{n-1}}\right]
\end{aligned}
$$

satisfied the above conditions and in this case, we denote the group scheme by $\mathscr{E}_{n} p^{l}$.

\section{The Proof of Theorem 1}

In this section we give our proof of Theorem 1. Suppose $A$ is a commutative unitary ring of characteristic $p$. Let $\lambda_{1}, \lambda_{2}, \ldots, \lambda_{n}$ be elements of $A$ and $\mathscr{E}_{n}$ be a 
group scheme defined in section 4.1 and $\hat{\mathscr{E}}_{n}$ be the formal completion of $\mathscr{E}_{n}$ along the zero section. We can easily see that the map

$$
\psi^{(l)}: \hat{\mathscr{E}}_{n} \rightarrow \hat{\mathscr{E}}_{n}^{p^{l}} ; \quad\left(x_{0}, \ldots, x_{n-1}\right) \mapsto\left(x_{0}^{p^{l}}, \ldots, x_{n-1}^{p^{l}}\right),
$$

is a homomorphism and the kernel of this isogeny is given by

$$
\begin{aligned}
N_{l}=\operatorname{Ker} \psi^{(l)} & =\operatorname{Spf} A\left[\left[X_{0}, X_{1}, \ldots, X_{n-1}\right]\right] /\left(X_{0}^{p^{l}}, X_{1}^{p^{l}}, \ldots, X_{n-1}^{p^{l}}\right) \\
& =\operatorname{Spec} A\left[X_{0}, X_{1}, \ldots, X_{n-1}\right] /\left(X_{0}^{p^{l}}, X_{1}^{p^{l}}, \ldots, X_{n-1}^{p^{l}}\right)
\end{aligned}
$$

since $X_{0}, X_{1}, \ldots, X_{n-1}$ are nilpotents in the coordinate ring of $N_{l}$. The following exact sequence is induced by the homomorphism $\psi^{(l)}$;

$$
0 \longrightarrow N_{l} \stackrel{l}{\longrightarrow} \hat{\mathscr{E}}_{n} \stackrel{\psi^{(l)}}{\longrightarrow} \hat{\mathscr{E}}_{n}^{p^{l}} \longrightarrow 0
$$

where $l$ is the canonical inclusion. This exact sequence (1) deduces the following long exact sequence;

$$
\begin{gathered}
0 \longrightarrow \operatorname{Hom}\left(\hat{\mathscr{E}} p^{l}, \hat{\mathbf{G}}_{m, A}\right) \stackrel{\psi^{(l)^{*}}}{\longrightarrow} \operatorname{Hom}\left(\hat{\mathscr{E}}_{n}, \hat{\mathbf{G}}_{m, A}\right) \stackrel{(l)^{*}}{\longrightarrow} \operatorname{Hom}\left(N_{l}, \hat{\mathbf{G}}_{m, A}\right) \\
\stackrel{\partial}{\longrightarrow} \operatorname{Ext}^{1}\left(\hat{\mathscr{E}}_{n}^{p^{l}}, \hat{\mathbf{G}}_{m, A}\right) \stackrel{\psi^{(l)^{*}}}{\longrightarrow} \operatorname{Ext}^{1}\left(\hat{\mathscr{E}}_{n}, \hat{\mathbf{G}}_{m, A}\right) \longrightarrow \\
\longrightarrow
\end{gathered}
$$

As a consequance of the argument in the proofs of Lemma 4 and Lemma 5, we will see that in the exact sequences, we can replace $\operatorname{Ext}^{1}\left(\hat{\mathscr{E}} p_{n}^{l}, \hat{\mathbf{G}}_{m, A}\right)$ and $\operatorname{Ext}^{1}\left(\hat{\mathscr{E}}_{n}, \hat{\mathbf{G}}_{m, A}\right)$ with the Hochschild cohomology groups $H_{0}^{2}\left(\hat{\mathscr{E}}_{n}^{p^{l}}, \hat{\mathbf{G}}_{m, A}\right)$ and $H_{0}^{2}\left(\hat{\mathscr{E}}_{n}, \hat{\mathbf{G}}_{m, A}\right)$ respectively. Here $H_{0}^{2}(\hat{\boldsymbol{G}}, \hat{H})$ denote the Hochschid cohomology group consisting of symmetric 2-cocycles of $\hat{G}$ with coefficients in $\hat{H}$ for formal group schemes $G$ and $H$. (c.f. [DG, II.2 and Chap III.6].) Therfore we have the following exact sequence;

$$
\begin{gathered}
0 \longrightarrow \operatorname{Hom}\left(\hat{\mathscr{E}}_{n}^{p^{l}}, \hat{\mathbf{G}}_{m, A}\right) \stackrel{\psi^{(l)^{*}}}{\longrightarrow} \operatorname{Hom}\left(\hat{\mathscr{E}}_{n}, \hat{\mathbf{G}}_{m, A}\right) \stackrel{(l)^{*}}{\longrightarrow} \operatorname{Hom}\left(N_{l}, \hat{\mathbf{G}}_{m, A}\right) \\
\stackrel{\partial}{\longrightarrow} H_{0}^{2}\left(\hat{\mathscr{E}}_{n}^{p^{l}}, \hat{\mathbf{G}}_{m, A}\right) \stackrel{\psi^{(l)^{*}}}{\longrightarrow} H_{0}^{2}\left(\hat{\mathscr{E}}_{n}, \hat{\mathbf{G}}_{m, A}\right) \stackrel{ }{\longrightarrow} \quad \ldots .
\end{gathered}
$$

On the other hand, as in the case $n$ of [SS2, Theorem 5.1], the following morphisms are isomorphic;

$$
\begin{array}{r}
\xi_{0}^{n}: \operatorname{Ker}\left[U^{n}: W(A)^{n} \rightarrow W(A)^{n}\right] \rightarrow \operatorname{Hom}\left(\hat{\mathscr{E}}_{n}, \hat{\mathbf{G}}_{m, A}\right) \\
\overline{\boldsymbol{v}}^{n}=\left(\overline{\boldsymbol{v}}_{i}^{n}\right) \mapsto E_{p}\left(\overline{\boldsymbol{v}}^{n},\left(\lambda_{i}\right) ; \mathbf{X}\right) \\
\xi_{1}^{n}: \operatorname{Coker}\left[U^{n}: W(A)^{n} \rightarrow W(A)^{n}\right] \rightarrow H_{0}^{2}\left(\hat{\mathscr{E}}_{n}, \hat{\mathbf{G}}_{m, A}\right) ; \\
\overline{\boldsymbol{w}}^{n}=\left(\overline{\boldsymbol{w}}_{i}^{n}\right) \mapsto F_{p}\left(\overline{\boldsymbol{w}}^{n},\left(\lambda_{i}\right) ; \mathbf{X}, \mathbf{Y}\right) .
\end{array}
$$


We put

$$
U^{n}:=\left(\begin{array}{ccccc}
F^{\left(\lambda_{1}\right)} & -T_{\boldsymbol{b}_{1}^{2}} & \cdots & \cdots & -T_{\boldsymbol{b}_{1}^{n}} \\
0 & F^{\left(\lambda_{2}\right)} & -T_{\boldsymbol{b}_{2}^{3}} & \cdots & -T_{\boldsymbol{b}_{2}^{n}} \\
0 & \cdots & & & -T_{\boldsymbol{b}_{n-1}^{n}} \\
0 & & \cdots & & F^{\left(\lambda_{n}\right)}
\end{array}\right)
$$

and

$$
\left(U^{n}\right)^{\prime}:=\left(\begin{array}{ccccc}
F^{\left(\lambda_{1}^{p^{l}}\right)} & -T_{\mathbf{B}_{1}^{2}} & \cdots & \cdots & -T_{\mathbf{B}_{1}^{n}} \\
0 & F^{\left(\lambda_{2}^{p^{l}}\right)} & -T_{\mathbf{B}_{2}^{3}} & \cdots & -T_{\mathbf{B}_{2}^{n}} \\
0 & \cdots & & & -T_{\mathbf{B}_{n-1}^{n}} \\
0 & & \ldots & & F^{\left(\lambda_{n}^{p^{l}}\right)}
\end{array}\right)
$$

where $\boldsymbol{b}_{i}^{j}$ and $\mathbf{B}_{i}^{j}$ (see section 4 ) are Witt vectors. We consider the following diagram;

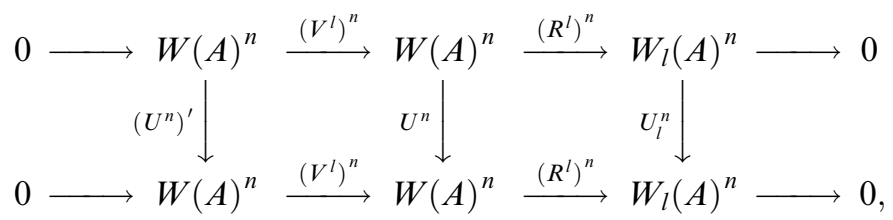

where $U_{l}^{n}$ is the restriction morphism of $U^{n}$ to $W_{l}(A)^{n}$. Then we have the commutativity of this diagram.

Proposition 1. The diagram $(*)$ is commutative.

LEMMA 1. $\quad T_{\boldsymbol{b}_{1}^{2}} V^{l}=V^{l} T_{\mathbf{B}_{1}^{2}}$.

Proof. This follows from [AA, Sublemma 1].

By using this lemma, we get

$$
\begin{aligned}
\mathbf{B}_{j}^{k+1} & =\frac{1}{\lambda_{k+1}^{p^{l}}}\left(F^{\left(\lambda_{j}^{p^{l}}\right)} \mathbf{A}_{j}^{k}-\sum_{l=j+1}^{k} T_{\mathbf{B}_{j}^{l}} \mathbf{A}_{l}^{k}\right) \\
& =\left(\frac{1}{\lambda_{k+1}}\left(F^{\left(\lambda_{j}\right)} \boldsymbol{a}_{j}^{k}-\sum_{l=j+1}^{k} T_{\boldsymbol{b}_{j}^{l}} \boldsymbol{a}_{l}^{k}\right)\right)^{p^{l}} \\
& =\boldsymbol{b}_{i}^{j\left(p^{l}\right)} \quad 1 \leqq j \leqq k-1 .
\end{aligned}
$$

First we will check the equality $U^{n} \circ\left(V^{l}\right)^{n}=\left(V^{l}\right)^{n} \circ\left(U^{n}\right)^{\prime}$. 
In fact for $\boldsymbol{v}^{n}=\left(\boldsymbol{v}_{i}^{n}\right)_{1 \leqq i \leqq n} \in W(A)^{n}$, we have

$$
\begin{aligned}
U^{n} \circ\left(V^{l}\right)^{n}\left(\boldsymbol{v}^{n}\right) & =\left(\begin{array}{ccccc}
F^{\left(\lambda_{1}\right)} V^{l} \boldsymbol{v}_{1}^{n} & -T_{\boldsymbol{b}_{1}^{2}} V^{l} \boldsymbol{v}_{2}^{n} & \cdots & \cdots & -T_{\boldsymbol{b}_{1}^{n}} V^{l} \boldsymbol{v}_{n}^{n} \\
0 & F^{\left(\lambda_{2}\right)} V^{l} \boldsymbol{v}_{2}^{n} & -T_{\boldsymbol{b}_{2}^{3}} V^{l} \boldsymbol{v}_{3}^{n} & \cdots & -T_{\boldsymbol{b}_{2}^{n}} V^{l} \boldsymbol{v}_{n}^{n} \\
0 & \cdots & & & -T_{\boldsymbol{b}_{n-1}^{n}} V^{l} \boldsymbol{v}_{n}^{n} \\
0 & & \ldots & & F^{\left(\lambda_{n}\right)} V^{l} \boldsymbol{v}_{n}^{n}
\end{array}\right) \\
& =\left(\begin{array}{cccccc}
V^{l} F^{\left(\lambda_{1}^{p^{l}}\right)} \boldsymbol{v}_{1}^{n} & -V^{l} T_{\mathbf{B}_{1}^{2}} \boldsymbol{v}_{2}^{n} & \cdots & \cdots & -V^{l} T_{\mathbf{B}_{1}^{n}} \boldsymbol{v}_{n}^{n} \\
0 & V^{l} F^{\left.\left(\lambda_{2}^{l}\right)^{l}\right)} \boldsymbol{v}_{2}^{n} & -V^{l} T_{\mathbf{B}_{2}^{3}} \boldsymbol{v}_{3}^{n} & \cdots & -V^{l} T_{\mathbf{B}_{2}^{n}} \boldsymbol{v}_{n}^{n} \\
0 & \cdots & & & -V^{l} T_{\mathbf{B}_{n-1}^{n} \boldsymbol{v}_{n}^{n}} \\
0 & & & & & V^{l} F^{\left(\lambda_{n}^{p^{l}}\right)} \boldsymbol{v}_{n}^{n}
\end{array}\right) \\
& =\left(V^{l}\right)^{n} \circ\left(U^{n}\right)^{\prime}\left(\boldsymbol{v}^{n}\right) . \\
\square &
\end{aligned}
$$

The next equality $U_{l}^{n} \circ\left(R_{l}\right)^{n}=\left(R_{l}\right)^{n} \circ U^{n}$ is a direct conseqence of the definition of $U_{l}^{n}$. The exactness of the horizontal sequences are obvious. By applying the snake lemma to $(*)$, we have the following exact sequence;

$$
\begin{aligned}
& 0 \longrightarrow \operatorname{Ker}\left(U^{n}\right)^{\prime} \stackrel{\left(V^{l}\right)^{n}}{\longrightarrow} \operatorname{Ker} U^{n} \stackrel{\left(R_{l}\right)^{n}}{\longrightarrow} \text { Ker } U_{l}^{n} \\
& \stackrel{\partial}{\longrightarrow} \operatorname{Coker}\left(U^{n}\right)^{\prime} \stackrel{\left(V^{l}\right)^{n}}{\longrightarrow} \operatorname{Coker} U^{n} \stackrel{\left(R_{l}\right)^{n}}{\longrightarrow} \text { Coker } U_{l}^{n} \longrightarrow 0 .
\end{aligned}
$$

Then, we can combine the exact sequence (3), (6) and the isomorphisms (4), (5) we have the following diagram in which the two horizontal sequences are exact, and vertical morphisms except for $\phi$ are isomorphisms;

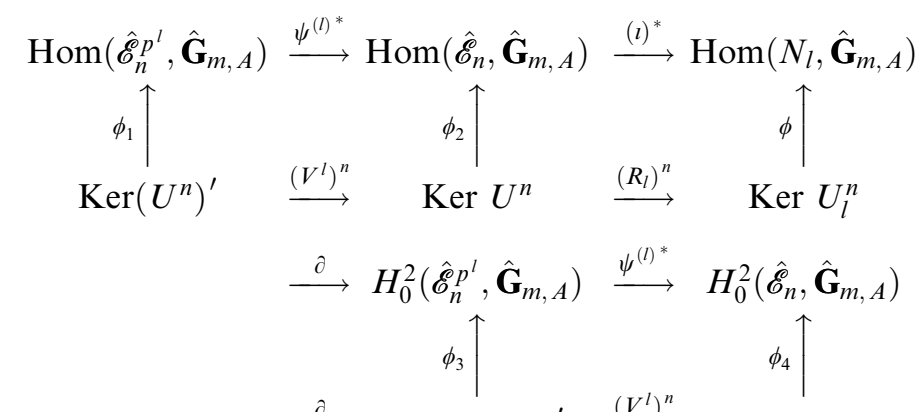

$\stackrel{\partial}{\longrightarrow} \operatorname{Coker}\left(U^{n}\right)^{\prime} \quad \stackrel{\left(V^{l}\right)^{n}}{\longrightarrow} \quad$ Coker $U^{n} \quad \stackrel{\left(R_{l}\right)^{n}}{\longrightarrow}$ Coker $U_{l}^{n}$.

Here, $\phi$ is the composite map $(l)^{*} \circ \xi_{0}^{n}$ of the morphism $(l)^{*}$ in (3) with the isomorphism $\xi_{0}^{n}$ in (4). If the diagram (7) is proved to be true, we get the isomorphism $\phi: \operatorname{Ker}\left[U_{l}^{n}: W_{l}(A)^{n} \rightarrow W_{l}(A)^{n}\right] \simeq \operatorname{Hom}\left(N_{l}, \hat{\mathbf{G}}_{m, A}\right)$ by the five lemma. So we obtain the Theorem 1 . Next we will check the commutativity of (7). 
Lemma 2. $\left(\psi^{(l)}\right)^{*} \circ \phi_{1}=\phi_{2} \circ\left(V^{l}\right)^{n}$.

Proof. For $\left(\boldsymbol{v}^{n}\right)=\left(\boldsymbol{v}_{\boldsymbol{i}}^{\boldsymbol{n}}\right)_{1 \leqq \boldsymbol{i} \leqq \boldsymbol{n}}$, we have

$$
\begin{aligned}
E_{p}\left(\left(\boldsymbol{v}^{n}\right),\left(\lambda_{i}^{p^{l}}\right)_{1 \leqq i \leqq n} ;\left(x_{i-1}\right)_{1 \leqq i \leqq n}\right) & =\prod_{i=1}^{n} E_{p}\left(\boldsymbol{v}_{i}^{n},\left(\lambda_{i}^{p^{l}}\right) ; \frac{x_{i-1}^{p^{l}}}{D_{i}^{\prime}\left(x_{0}^{p^{l}}, x_{1}^{p^{l}}, \ldots, x_{n-2}^{p^{l}}\right)}\right) \\
& =\prod_{i=1}^{n} E_{p}\left(\boldsymbol{v}_{i}^{n},\left(\lambda_{i}^{p^{l}}\right) ;\left(\frac{x_{i-1}}{D_{i}^{\prime}\left(x_{0}, x_{1}, \ldots, x_{n-2}\right)}\right)^{p^{l}}\right) \\
& =\prod_{i=1}^{n} E_{p}\left(V^{l} \boldsymbol{v}_{i}^{n},\left(\lambda_{i}\right) ; \frac{x_{i-1}}{D_{i}\left(x_{0}, x_{1}, \ldots, x_{n-2}\right)}\right) \\
& =E_{p}\left(V^{l}\left(\boldsymbol{v}^{n}\right),\left(\lambda_{i}\right)_{1 \leqq i \leqq n} ;\left(x_{i-1}\right)_{1 \leqq i \leqq n}\right) .
\end{aligned}
$$

These equalities means our assertions.

LEMMA 3. $(l)^{*} \circ \phi_{2}=\phi \circ\left(R_{l}\right)^{n}$.

Proof. This follows immediately from the definitions of $\phi$ and $(l)^{*}$.

LEMMA 4. $\partial \circ \phi=\phi_{3} \circ \partial$.

Proof. For $\left(R_{l}\right)^{n} \boldsymbol{v}^{n}=\left(R_{l} \boldsymbol{v}_{i}^{n}\right)_{1 \leqq i \leqq n} \in \operatorname{Ker} U_{l}^{n}$, we caculate $\partial E_{p}\left(\left(R_{l}\right)^{n} \boldsymbol{v}^{n}\right.$, $\left.\left(\lambda_{i}\right)_{1 \leqq i \leqq n} ;\left(x_{i-1}\right)_{1 \leqq i \leqq n}\right) \in \operatorname{Ker} U_{l}^{n}$ on the fibre product $\hat{\mathbf{G}}_{m, A} \times \hat{\mathscr{E}}_{n}^{p^{l}}$ where the following diagram is commutative;

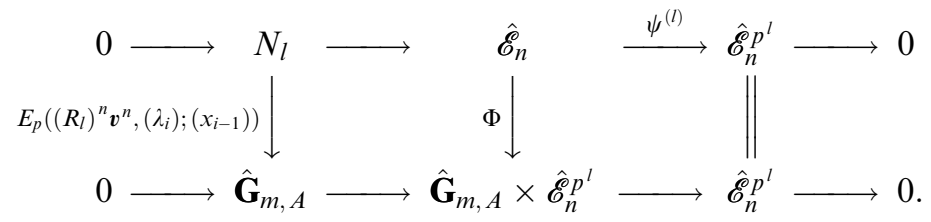

By the above condition, we get $\Phi$ as the following map;

$$
\Phi: \hat{\mathscr{E}}_{n} \rightarrow \hat{\mathbf{G}}_{m, A} \times \hat{\mathscr{E}}_{n}^{p^{l}} ; \quad\left(x_{i-1}\right) \mapsto\left(E_{p}\left(\left(\boldsymbol{v}_{i}^{n}\right),\left(\lambda_{i}\right) ;\left(x_{i-1}\right)\right), \psi^{(l)}\left(\left(x_{i-1}\right)\right)\right)
$$

so we must endow $\hat{\mathbf{G}}_{m, A} \times \hat{\mathscr{E}}_{n}^{p^{l}}$ with a group scheme structrures so that $\Phi$ is a homomorphism. This means the equality;

$$
\Phi\left(\left(x_{i-1}\right),\left(y_{i-1}\right)\right)=\Phi\left(\left(x_{i-1}\right)\right) \cdot \Phi\left(\left(y_{i-1}\right)\right), \quad\left(x_{i-1}\right),\left(y_{i-1}\right) \in \hat{\mathscr{E}}_{n},
$$


where

$$
\begin{gathered}
\Phi\left(\left(x_{i-1}\right) \cdot\left(y_{i-1}\right)\right)=\left(E_{p}\left(\left(\boldsymbol{v}_{i}^{n}\right),\left(\lambda_{i}\right) ;\left(x_{i-1}\right) \cdot\left(y_{i-1}\right)\right), \psi^{(l)}\left(\left(x_{i-1}\right) \cdot \psi^{(l)}\left(y_{i-1}\right)\right)\right), \\
\Phi\left(\left(x_{i-1}\right)\right) \cdot \Phi\left(\left(y_{i-1}\right)\right)=\left(E_{p}\left(\left(\boldsymbol{v}_{i}^{n}\right),\left(\lambda_{i}\right) ;\left(x_{i-1}\right)\right), \psi^{(l)}\left(\left(x_{i-1}\right)\right)\right) \\
\cdot\left(E_{p}\left(\left(\boldsymbol{v}_{i}^{n}\right),\left(\lambda_{i}\right) ;\left(y_{i-1}\right)\right), \psi^{(l)}\left(\left(y_{i-1}\right)\right)\right) .
\end{gathered}
$$

For elements $\left(t_{1},\left(z_{i-1}\right)\right),\left(t_{2},\left(w_{i-1}\right)\right)$ of $\hat{\mathbf{G}}_{m, A} \times \hat{\mathscr{E}}_{n}^{p^{l}}$, we choose the inverse images $\left(x_{i-1}\right)$ and $\left(y_{i-1}\right)$ of $\left(z_{i-1}\right)$ and $\left(w_{i-1}\right)$ with respect to the $\psi^{(l)}$, respectively. Then the group sturucture of $\hat{\mathbf{G}}_{m, A} \times \hat{\mathscr{E}}_{n}^{p^{l}}$ should be given by

$$
\begin{aligned}
& \left(t_{1},\left(z_{i-1}\right)\right) \cdot\left(t_{2},\left(w_{i-1}\right)\right) \\
& \quad=\left(t_{1} t_{2} \cdot \frac{E_{p}\left(\left(\boldsymbol{v}_{i}^{n}\right),\left(\lambda_{i}\right) ;\left(x_{i-1}\right) \cdot\left(y_{i-1}\right)\right)}{\left(E_{p}\left(\left(\boldsymbol{v}_{i}^{n}\right),\left(\lambda_{i}\right) ;\left(x_{i-1}\right)\right)\right) \cdot\left(E_{p}\left(\left(\boldsymbol{v}_{i}^{n}\right),\left(\lambda_{i}\right) ;\left(y_{i-1}\right)\right)\right)},\left(z_{i-1}\right) \cdot\left(w_{i-1}\right)\right) \\
& \quad=F_{p}\left(U^{n}\left(\boldsymbol{v}_{i}^{n}\right),\left(\lambda_{i}\right) ;\left(x_{i-1}\right),\left(y_{i-1}\right)\right) .
\end{aligned}
$$

Next we must show the following equation;

$$
F_{p}\left(U^{n}\left(\boldsymbol{v}_{i}^{n}\right),\left(\lambda_{i}\right) ;\left(x_{i-1}\right),\left(y_{i-1}\right)\right)=F_{p}\left(\left(\boldsymbol{w}_{i}^{n}\right),\left(\lambda_{i}\right) ;\left(x_{i-1}\right),\left(y_{i-1}\right)\right)
$$$$
\text { (note that } \left.\left(V^{l}\right)^{n}\left(\boldsymbol{w}_{i}^{n}\right)=U^{n}\left(\boldsymbol{v}_{i}^{n}\right)\right) \text {. }
$$

$$
\begin{aligned}
F_{p}\left(U^{n}\right. & \left.\left(\boldsymbol{v}_{i}^{n}\right),\left(\lambda_{i}\right) ;\left(x_{i-1}\right),\left(y_{i-1}\right)\right) \\
= & \prod_{i=1}^{n} F_{p}\left(V^{l} \boldsymbol{w}_{i}^{n}, \lambda_{i} ; \frac{x_{i-1}}{D_{i-1}\left(x_{0}, \ldots, x_{n-2}\right)}, \frac{y_{i-1}}{D_{i-1}\left(y_{0}, \ldots, y_{n-2}\right)}\right) \\
& \times \prod_{i=1}^{n} F_{p}\left(V^{l} \boldsymbol{w}_{i}^{n}, \lambda_{i} ; H_{i-1}, \frac{x_{i-1}}{D_{i-1}\left(x_{0}, \ldots, x_{n-2}\right)}+\frac{y_{i-1}}{D_{i-1}\left(y_{0}, \ldots, y_{n-2}\right)}\right) \\
& \times \prod_{p} G_{p}\left(V^{l} \boldsymbol{w}_{i}^{n}, \lambda_{i} ; F^{i-1}\right)^{-1} \\
= & \prod_{i=1}^{n} F_{p}\left(\boldsymbol{w}_{i}^{n}, \lambda_{i}^{p^{l}} ;\left(\frac{x_{i-1}}{D_{i-1}\left(x_{0}, \ldots, x_{n-2}\right)}\right)^{p^{l}}\right) \\
& \times \prod_{i=1}^{n} F_{p}\left(V^{l} \boldsymbol{w}_{i}^{n}, \lambda_{i}^{p^{l}} ; H_{i-1}^{\left(p^{l}\right)},\left(\frac{y_{i-1}\left(y_{0}, \ldots, y_{n-2}\right.}{D_{i-1}\left(x_{0}, \ldots, x_{n-2}\right)} \dot{+} \frac{y_{i-1}}{D_{i-1}\left(y_{0}, \ldots, y_{n-2}\right)}\right)^{p^{l}}\right) \\
& \times \prod G_{p}\left(V^{l} \boldsymbol{w}_{i}^{n}, \lambda_{i}^{p^{l}} ;\left(F^{(i-1)}\right)^{\left(p^{l}\right)}\right)^{-1} \\
= & F_{p}\left(U^{n}\left(\boldsymbol{w}_{i}^{n}\right),\left(\lambda_{i}^{p^{l}}\right) ;\left(x_{i-1}^{p^{l}}\right),\left(y_{i-1}^{p^{l}}\right)\right) .
\end{aligned}
$$

This means our assertion. 
LEMMA 5. $\psi^{(l)} \circ \phi_{3}=\phi_{4} \circ\left(V^{l}\right)^{n}$.

Proof. For $\left(\boldsymbol{v}^{n}\right) \in \operatorname{Coker}\left(U^{n}\right)^{\prime}$, we can determine the direct image;

$$
\left(\psi^{(l)}\right)^{*} F_{p}\left(\left(\boldsymbol{v}_{i}^{n}\right),\left(\lambda_{i}^{p^{l}}\right) ;\left(z_{i-1}\right),\left(w_{i-1}\right)\right)
$$

on the fibre product $\hat{\mathbf{G}}_{m, A} \times \hat{\mathscr{E}}_{n}$, so we look at the following commutative diagram;

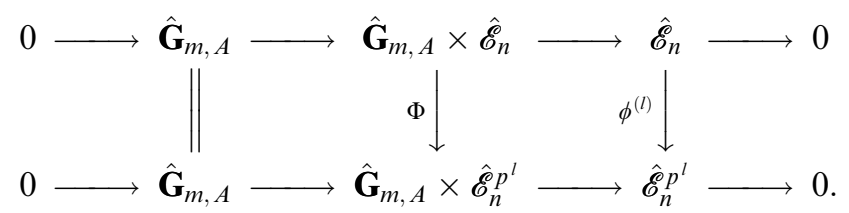

By the condition of diagram (9), we have a map $\Phi$ given by

$$
\Phi: \hat{\mathbf{G}}_{m, A} \times \hat{\mathscr{E}}_{n} \rightarrow \hat{\mathbf{G}}_{m, A} \times \hat{\mathscr{E}}_{n}^{p^{l}} ; \quad\left(t,\left(x_{i-1}\right)\right) \mapsto\left(t, \phi^{(l)}\left(\left(x_{i-1}\right)\right)\right) .
$$

We endow $\hat{\mathbf{G}}_{m, A} \times \hat{\mathscr{E}}_{n}$ with a group scheme structure so that $\Phi$ becomes a homomorphism. Let $\left(t_{1},\left(x_{i-1}\right)\right)$ and $\left(t_{2},\left(y_{i-1}\right)\right)$ be local sections in $\hat{\mathbf{G}}_{m, A} \times \hat{\mathscr{E}}_{n}$. If the product $\left(t_{1},\left(x_{i-1}\right)\right) \cdot\left(t_{2},\left(y_{i-1}\right)\right)$ is expressed as $\left(t_{1},\left(x_{i-1}\right)\right) \cdot\left(t_{2},\left(y_{i-1}\right)\right)=$ $\left(t_{1} t_{2} G\left(\left(x_{i-1}\right),\left(y_{i-1}\right)\right),\left(x_{i-1}\right) \cdot\left(y_{i-1}\right)\right)$ where $G\left(\left(x_{i-1}\right),\left(y_{i-1}\right)\right)$ is a cocycle on $\hat{\mathbf{G}}_{m, A} \times \hat{\mathscr{E}}_{n}$. Then we have the following equation;

$$
\begin{aligned}
\Phi\left(\left(t_{1},\left(x_{i-1}\right)\right) \cdot\left(t_{2},\left(y_{i-1}\right)\right)\right) & =\Phi\left(t_{1} t_{2} G\left(\left(x_{i-1}\right),\left(y_{i-1}\right)\right),\left(x_{i-1}\right) \cdot\left(y_{i-1}\right)\right) \\
& =\left(t_{1} t_{2} G\left(\left(x_{i-1}\right),\left(y_{i-1}\right)\right), \phi^{(l)}\left(x_{i-1}\right) \cdot \phi^{(l)}\left(y_{i-1}\right)\right) .
\end{aligned}
$$

On the other hand, we have

$$
\begin{aligned}
& \Phi\left(\left(t_{1},\left(x_{i-1}\right)\right) \cdot \Phi\left(\left(t_{2},\left(y_{i-1}\right)\right)\right)\right. \\
& \quad=\left(t_{1}, \phi^{(l)}\left(x_{i-1}\right)\right) \cdot\left(t_{2}, \phi^{(l)}\left(y_{i-1}\right)\right) \\
& \quad=\left(t_{1} t_{2} F_{p}\left(\left(\boldsymbol{v}^{n}\right),\left(\lambda_{i}^{p^{l}}\right) ; \phi^{(l)}\left(x_{i-1}\right), \phi^{(l)}\left(y_{i-1}\right)\right), \phi^{(l)}\left(x_{i-1}\right) \cdot \phi^{(l)}\left(y_{i-1}\right)\right) .
\end{aligned}
$$

Hence it is necessarly to have the following condition that $\Phi$ is a homomorphism;

$$
G\left(\left(x_{i-1}\right),\left(y_{i-1}\right)\right)=F_{p}\left(\left(\boldsymbol{v}^{n}\right),\left(\lambda_{i}^{p^{l}}\right) ; \phi^{(l)}\left(x_{i-1}\right), \phi^{(l)}\left(x_{i-1}\right)\right) .
$$

We'll show the next equation to prove this;

$$
F_{p}\left(\left(\boldsymbol{v}^{n}\right),\left(\lambda_{i}^{p^{l}}\right) ; \phi^{(l)}\left(x_{i-1}\right), \phi^{(l)}\left(x_{i-1}\right)\right)=F_{p}\left(V^{l}\left(\boldsymbol{v}^{n}\right),\left(\lambda_{i}\right) ; \phi^{(l)}\left(x_{i-1}\right), \phi^{(l)}\left(y_{i-1}\right)\right) .
$$

(But it has already proved in [AA, lemma 3].) 


\section{The Kernel of the Type $F^{n}+a_{1} F^{n-1}+\cdots+a_{n}$}

In the previous paper $[\mathrm{AA}]$, we can construct the case where the endomorphism $T_{\boldsymbol{b}}$ becomes the identity map. Taking the similar method, we give such a description in the generalized situation when $n$ is arbitrary, $T_{\boldsymbol{b}_{1}^{2}}, T_{\boldsymbol{b}_{2}^{3}}, \ldots$, and $T_{\boldsymbol{b}_{n-1}^{n}}$ are identity maps and the other $T_{\boldsymbol{b}_{i}^{j}}$ 's are zero maps. So we get

$$
U^{n}=\left(\begin{array}{ccccc}
F^{\left(\lambda_{1}\right)} & -1 & \cdots & \cdots & 0 \\
0 & F^{\left(\lambda_{2}\right)} & -1 & \cdots & 0 \\
0 & \cdots & & & -1 \\
0 & & \cdots & & F^{\left(\lambda_{n}\right)}
\end{array}\right) .
$$

Let $\left(\boldsymbol{v}_{l}^{n}\right)$ be the element of $\operatorname{Ker} U_{l}^{n}$, we have following equations;

$$
\begin{aligned}
& F^{\left(\lambda_{1}\right)} \boldsymbol{v}_{l}^{1}-\boldsymbol{v}_{l}^{2}=0 \\
& F^{\left(\lambda_{2}\right)} \boldsymbol{v}_{l}^{2}-\boldsymbol{v}_{l}^{3}=0 \\
& \vdots \\
& F^{\left(\lambda_{n-1}\right)} \boldsymbol{v}_{l}^{n-1}-\boldsymbol{v}_{l}^{n}=0 \\
& F^{\left(\lambda_{n}\right)} \boldsymbol{v}_{l}^{n}=0 .
\end{aligned}
$$

Hence we have the next equations;

$$
\begin{aligned}
\boldsymbol{v}_{l}^{2} & =F^{\left(\lambda_{1}\right)} \boldsymbol{v}_{l}^{1} \\
\boldsymbol{v}_{l}^{3} & =F^{\left(\lambda_{2}\right)} \boldsymbol{v}_{l}^{2}=F^{\left(\lambda_{2}\right)} F^{\left(\lambda_{1}\right)} \boldsymbol{v}_{l}^{1} \\
& \vdots \\
\boldsymbol{v}_{l}^{n} & =F^{\left(\lambda_{n-1}\right)} F^{\left(\lambda_{n-2}\right)} \cdots F^{\left(\lambda_{1}\right)} \boldsymbol{v}_{l}^{1} \\
0 & =F^{\left(\lambda_{n}\right)} \boldsymbol{v}_{l}^{n}=F^{\left(\lambda_{n}\right)} F^{\left(\lambda_{n-1}\right)} \cdots F^{\left(\lambda_{1}\right)} \boldsymbol{v}_{l}^{1} .
\end{aligned}
$$

Therefore in this case we have the canonical isomorphism;

$$
\operatorname{Ker}\left[U_{l}^{n}: W_{l}^{n} \rightarrow W_{l}^{n}\right] \simeq \operatorname{Ker}\left[F^{\left(\lambda_{n}\right)} F^{\left(\lambda_{n-1}\right)} \cdots F^{\left(\lambda_{1}\right)}: W_{l} \rightarrow W_{l}\right]
$$

Moreover $F^{\left(\lambda_{n}\right)} F^{\left(\lambda_{n-1}\right)} \cdots F^{\left(\lambda_{1}\right)}$ is given the following polynomial in $F$;

$$
F^{\left(\lambda_{n}\right)} F^{\left(\lambda_{n-1}\right)} \cdots F^{\left(\lambda_{1}\right)}=F^{n}+\boldsymbol{a}_{1} F^{n-1}+\cdots+\boldsymbol{a}_{n},
$$


where the Witt vectors $\boldsymbol{a}_{k}$ 's are given by;

$$
\boldsymbol{a}_{k}=\sum_{n \geq i_{1}>i_{2} \cdots>i_{k} \geq 1}(-1)^{k}\left[\prod_{j=1}^{k} \lambda_{i_{k}}^{(p-1) p^{n-i_{j}-(j-1)}}\right] \quad k=1, \ldots, n .
$$

\section{References}

[AA] N. Aki, M. Amano, On the Cartier Duality of Certain Finite Group Schemes of type $\left(p^{n}, p^{n}\right)$ Tsukuba J. Math., Vol. 34, No. 1, (2010), 31-46.

[A] M. Amano, On The Cartier Duaulity of Certain Finite Group Schemes of order $p^{n}$, Tokyo J. Math., Vol. 33, No. 1.

[DG] M. Demazure and P. Gabriel, Groupes algébriques, Tome 1, Masson-North-Holland, ParisAmsterdam, 1970.

[HZ] M. Hazewinkel, Formal groups and applications, Academic Press, New York, 1978.

[OT] Oort, F., Tate, J., Group schemes of prime order, Ann. Sci. École Norm. Sup. (4) 22, (1989), 345-375.

[SOS] T. Sekiguchi, F. Oort, N. Suwa, On the deformation of Artin-Schreier to Kummer, Ann. Sci. École Norm. Sup. (4) 22, (1989), 345-375.

[SS1] T. Sekiguchi, N. Suwa, A note on extentions of algebraic and formal groups IV, Tohoku Math. J., 53, (2001), 203-240.

[SS2] T. Sekiguchi, N. Suwa, On the unified Kummer-Artin-Schreier-Witt theory, Prépublication N.111, université de Bordeaux, (1999).

(Nobuhiro Aki)

Department of Mathematics

Chuo University

1-13-27 Kasuga, Bunkyo-Ku, Tokyo, 112-8551 Japan

E-mail address: s87201@gug.math.chuo-u.ac.jp 